\title{
Antibiotic resistance is an emerging threat to public health: an urgent call to action at the Antimicrobial Resistance Summit 2011
}

\author{
Thomas Gottlieb and Graeme R Nimmo
}

\section{A national interdisciplinary body is urgently needed to manage the looming antimicrobial resistance crisis}

T he introduction of antibiotics was one of the most important developments in modern medicine. Their availability has facilitated increasingly complex care and, not surprisingly, microbial resistance to antibiotics has been identified as one of the greatest threats to human health. A return to the "preantibiotic era" would render many routine infections untreatable and would seriously affect current practice in surgery, intensive care, organ transplantation, neonatology and cancer services through major increases in morbidity and mortality. The time to act is now - before we lose these "miracle" drugs for good.

\section{Preserving the "miracle" of antibiotics}

In response to a looming crisis in antimicrobial resistance, the Australasian Society for Infectious Diseases and the Australian Society for Antimicrobials convened the Antimicrobial Resistance Summit in Sydney on 7-8 February 2011. The meeting brought together an interdisciplinary group of experts from the medical, veterinary, agricultural, infection control and public health sectors to establish priorities and a joint plan for action, focusing on the key elements shown in Box 1.

\section{The threat of antibiotic overuse}

The keynote address at the Summit was given by Professor Otto Cars (Professor of Infectious Diseases, Uppsala University, Sweden, and Chair of STRAMA, the Swedish strategic program against antibiotic resistance). STRAMA, a government-funded body, has supervised a multidisciplinary effort to improve surveillance and infection prevention and promote rational antimicrobial usage. This has been very successful in reducing usage in Sweden. Professor Cars highlighted that the key factor contributing to the alarming resistance trend in bacteria is the indiscriminate use of antibiotics.

\section{An agenda for addressing antimicrobial resistance}

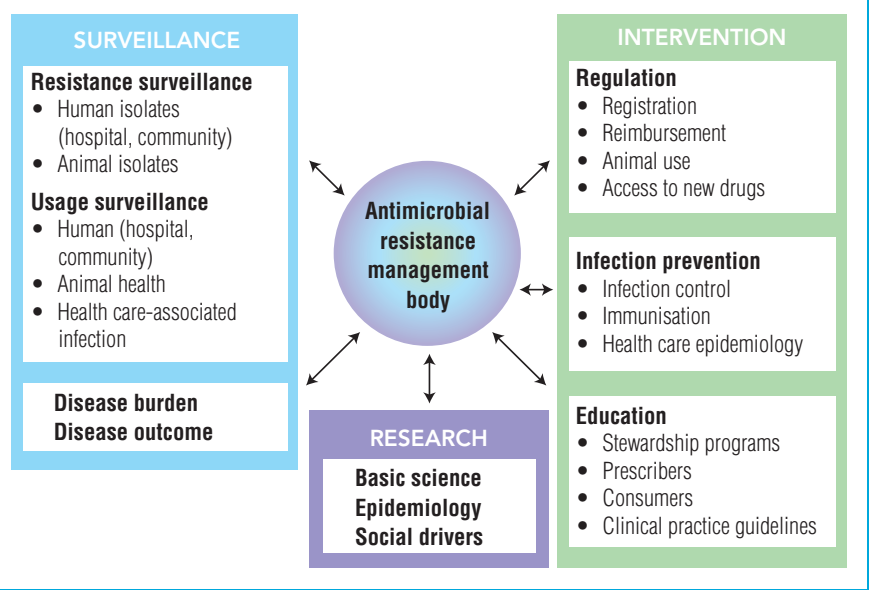

This is occurring in a number of settings including hospitals, general practice and veterinary medicine. Agricultural practices involving the use of antibiotics for prophylaxis and growth promotion rather than treatment also contribute to this problem. Repeated studies have demonstrated that a significant proportion of antibiotic use is unnecessary and that antibiotic use varies markedly between countries despite similar clinical outcomes. ${ }^{1}$

For many bacterial pathogens, resistance to last-line antibiotics, such as carbapenems, fluoroquinolones, glycopeptides and thirdgeneration cephalosporins, is now commonly found in Australian hospitals and, to an increasing extent, in the community. Examples include methicillin-resistant Staphylococcus aureus, multiresistant Streptococcus pneumoniae, vancomycin-resistant enterococci and multiresistant Escherichia coli. ${ }^{2}$ This growing ineffectiveness of once-reliable drugs has seen health care professionals increasingly turning to alternatives that are more toxic, more expensive and less likely to be orally available, putting increased pressure on a strained hospital system. In addition, compared with susceptible bacteria, antibiotic-resistant strains are associated with increased patient morbidity and mortality and increased costs of health care. $^{1}$

\section{A dwindling pipeline}

While the issue of antimicrobial resistance is not new, it has long been assumed that this problem would be overcome by the ongoing development of new compounds. However, there has been an alarming decline in antibiotic development over time, highlighting that this strategy cannot be relied upon. ${ }^{1}$

\section{The Antimicrobial Resistance Summit plan for action}

\section{Surveillance}

Measuring the extent of antimicrobial resistance in communityand health care-associated infections is crucial to defining the issue and measuring outcomes of any interventions designed to address it. While passive surveillance by electronic collation of routine susceptibility results can provide the broadest view of phenotypic changes, targeted surveillance by prospective studies of specific pathogens of interest provides details of newly emerging resistance mechanisms and strains. At present, the extent of antimicrobial resistance in Australia remains poorly defined. The current systems of data collection and collation vary between states and territories and there is limited coordination at a national level.

In addition to measuring antimicrobial resistance, it is important to understand antibiotic usage. Currently, the National Antimicrobial Utilisation Surveillance Program represents the only nationwide systematic surveillance of antibiotic usage, but it is based on voluntary data submitted from major hospitals, representing about $50 \%$ of Australian tertiary referral beds ${ }^{3}$ (Box 2). 


\section{Surveillance: key recommendations}

\section{Antimicrobial resistance surveillance}

- A comprehensive national surveillance system encompassing both passive and targeted components should be developed to monitor how much resistance is present, in which bacteria and where. This should include medical (hospital and community) and veterinary areas, as well as agriculture (including imported food).

- Priority should be given to staphylococci and Escherichia coli, which have the greatest impact on human health (emerging resistance in E. coli and other Gram-negative bacteria poses a major new threat).

- Methods used in resistance testing should be standardised wherever possible to enable comparison and pooling of data. In particular, minimum inhibitory concentration breakpoints need to be standardised.

\section{Antibiotic usage surveillance}

- A comprehensive national monitoring and audit system covering all areas of antibiotic usage should be established. This should include comprehensive surveillance of hospital usage (eg, by expanding the National Antimicrobial Utilisation Surveillance Program), representative sampling of community prescribing, and collating distribution data from agricultural antibiotic suppliers.

- Data on the appropriateness of usage should also be evaluated (using point-prevalence surveys comparing diagnosis with prescription).

- Voluntary identification of hospitals in surveillance programs is recommended to encourage benchmarking and transparency.

\section{Education and stewardship}

Education of the public as well as medical, veterinary and public health sectors is a crucial component in achieving judicious prescribing and dispensing of antibiotics. However, research has demonstrated that education campaigns and guidelines are ineffective unless they are combined with sustained interventions such as audit and feedback methods and/or a system where proactive steps are taken to assist prescribing and interventions are made to address poor performance. ${ }^{4}$ Antimicrobial stewardship acts at an organisational level and defines collective strategies to optimise antimicrobial prescribing through sustainable changes in practice. Further initiatives are required in the community, where the majority of human antibiotics are prescribed (Box 3).

\section{Infection prevention and control strategies}

Generic measures such as hand hygiene, avoidance of patient contamination during invasive procedures, environmental cleaning and disinfection, vaccination, and antibiotic stewardship are crucial in infection prevention and control. Surveillance is critical for determining the health care infection burden, identifying the people who are most at risk, and demonstrating the effectiveness of infection prevention or control interventions. To be effective, nationally coordinated surveillance is essential. Screening and effective isolation of patients carrying multiresistant organisms also has an important role ${ }^{1}$ (Box 4).

\section{Future research agenda}

While basic science research on microbiology is currently adequately funded by the National Health and Medical Research Council (NHMRC) and the Australian Research Council, a number of essential aspects required to combat antibiotic resistance do not find a ready place in existing project grant structures (Box 5).

\section{Education and stewardship: key recommendations}

- Educational initiatives need to define antimicrobial resistance as an urgent public health issue. The skills of social marketers and behavioural change experts should be used to drive a national campaign targeting both the public and prescribers. The National Prescribing Service (NPS) antibiotic educational campaign should also be reinstituted.

- A uniform national medical curriculum should be implemented that acknowledges overuse of antibiotics and embraces better stewardship, building on the NPS National Prescribing Curriculum.

- Stewardship, based on national antibiotic guidelines and local epidemiology, must be mandated in all hospitals within a quality framework based on audit and feedback.

- Effective stewardship requires a team-based approach including infection prevention units, microbiologists, pharmacists and clinicians. It must be strongly supported by senior hospital management and underpinned by effective use of information technology.

- Stewardship should also be extended beyond health care institutions to community care, long-term care facilities and nonmedical antibiotic use. A well resourced national body should be created to provide effective national coordination of educational activities and antibiotic stewardship.

- Recognising that antibiotic resistance does not respect international borders, Australia has an obligation to contribute to international programs to control antibiotic use and resistance.

4 Infection prevention and control strategies: key recommendations

- Improved training on strategies for infection prevention and control should be provided to health care workers.

- All major hospitals should have the epidemiological capacity to identify and investigate outbreaks of multiresistant organisms, and to monitor and respond to changing patterns of pathogens causing health care-associated infections.

- National evidence-based standards for multiresistant organism control in aged care facilities should be developed, implemented and robustly enforced and monitored.

- A national coordinating centre for the control of antimicrobial resistance is needed, with the authority and capacity to collect and analyse data on multiresistant organisms from all jurisdictions. This will facilitate timely, consistent and effective control of multiresistant organisms.

\section{Future research agenda: key recommendation}

- A new National Health and Medical Research Council funding call (similar to that for pandemic [H1N1] 2009 influenza) is needed for research on antimicrobial resistance and usage in Australia and its near neighbours. The key areas of focus should be:

$>$ epidemiology (in both human and animal settings)

$>$ effective interventions for the public sectors, focusing on education and behavioural change

$>$ effective interventions for the health care sector. 


\section{Regulation: key recommendations}

- Resistance risk assessments should be part of the regulatory process for bringing new antibiotics to market for both humans and animals.

- The Pharmaceutical Benefits Advisory Committee should consider resistance in the criteria for inclusion or restriction of antibiotics in the Pharmaceutical Benefits Scheme.

- Strategies should be implemented to fast-track important new antimicrobials through the regulatory approval system.

- Strategies should be implemented to enable the registration of non-commercial "orphan" drugs that have the potential to improve patient outcomes and reduce disease burden.

- Adopting an antibiotic importance rating system as regulatory policy should be considered.

\section{Regulation}

Implementing effective regulatory controls represents an important aspect of reducing indiscriminate use of antibiotics and keeping levels of antimicrobial resistance low in both human and animal settings (Box 6).

\section{An urgent call to action}

The threat of multiresistant bacteria is a critical public health issue that requires a coordinated, multifaceted response. To achieve this level of cooperation, we propose the creation of a national antimicrobial resistance management body comprising a wide range of stakeholders, including health care professionals, veterinarians, agriculturalists, pharmaceutical manufacturers, government, media representatives, consumers and other interested parties. The role of this body would include:

- implementing a comprehensive national resistance monitoring and audit system

- coordinating education and stewardship programs

- implementing infection prevention and control guidelines

- expanding funding to support research into all aspects of antibiotic resistance

- reviewing and upgrading the current regulatory system applying to antibiotics.

This strategy is recommended by the World Health Organization $^{1}$ and has been adopted by many other countries.

The scourge of antimicrobial resistance has increased inexorably over the years. We believe that the window for overcoming antimicrobial resistance is still open, but we must act decisively now - Australia cannot bury its head in the sand any longer and hope that the problem will just go away.

\section{Acknowledgements}

Summit Organising Committee Members: Dr Craig S Boutlis (Infectious Diseases Physician, Wollongong), Dr Kirsty Buising (Infectious Diseases Physician, Melbourne), Associate Professor Allen C Cheng (Infectious Diseases Physician, Melbourne), Professor Keryn Christiansen (Clinical Microbiologist, Perth), Professor Peter Collignon (Infectious Diseases Physician and Microbiologist, Canberra), Mr Geoffrey Coombs (Principal Scientist, Perth), Dr Marilyn Cruickshank (Policy Manager, Sydney), Professor Bart J Currie (Infectious Diseases Physician, Darwin), Ms Margaret Duguid (Pharmacist, Sydney), Dr John Ferguson (Infectious Diseases Physician and Microbiologist, Newcastle), Dr Rodney Givney (Microbiologist, Newcastle), Dr Ken Harvey (Clinical Microbiologist, Melbourne), Dr David Looke (Infectious Diseases Physician and Clinical Microbiologist, Brisbane), Dr Caroline Marshall (Infectious Diseases Physician, Melbourne), Dr Mary Murray (Global
Network Coordinator, ReAct Action on Antibiotic Resistance, Wee Jasper), Professor David L Paterson (Infectious Diseases Physician and Microbiologist, Brisbane), Professor Michael Richards (Infectious Diseases Physician, Melbourne), Professor John Turnidge (Infectious Disease Physician and Microbiologist, Adelaide). We thank Anne Burridge for help in drafting the manuscript and Nadine Giatras, Elizabeth Bennett and Petrana Lorenz at the Australasian Society for HIV Medicine for Summit organisation.

\section{Competing interests}

Thomas Gottlieb is President of the Australasian Society for Infectious Diseases. He has served on advisory boards for Novartis, Pfizer, AstraZeneca and Janssen-Cilag. Graeme Nimmo is President of the Australian Society for Antimicrobials. He has served on advisory boards for Novartis and Pfizer, and has received lecture fees from bioMerieux and reimbursement for travel and accommodation from Novartis, Pfizer and bioMerieux.

\section{Author details}

Thomas Gottlieb, MB BS, FRACP, FRCPA, Senior Physician, ${ }^{1}$ and Associate Professor ${ }^{2}$

Graeme R Nimmo, MD, FRCPA, FASM, State Director of Microbiology, ${ }^{3}$ and Professor ${ }^{4}$

1 Department of Infectious Diseases and Microbiology, Concord Hospital, Sydney, NSW.

2 Department of Medicine, University of Sydney, Sydney, NSW.

3 Pathology Queensland, Brisbane, QLD.

4 School of Medicine, Griffith University, Gold Coast, QLD.

Correspondence: thomas.gottlieb@sswahs.nsw.gov.au

\section{References}

1 World Health Organization Department of Communicable Disease Surveillance and Response. WHO global strategy for containment of antimicrobial resistance. Geneva: WHO, 2001. http://www.who.int/csr/ resources/publications/drugresist/WHO_CDS_CSR_DRS_2001_2_EN/ en/ (accessed Feb 2011).

2 Collignon P. Antimicrobial resistance. Med J Aust 2002; 177: 325-329.

3 National Antimicrobial Utilisation Surveillance Program. AUSP reports. Adelaide: NAUSP. http://www.health.sa.gov.au/INFECTIONCONTROL/ Default.aspx?tabid=199 (accessed Feb 2011)

4 Dellit TH, Owens RC, McGowan JE, et al. Infectious Diseases Society of America and the Society for Healthcare Epidemiology of America guidelines for developing an institutional program to enhance antimicrobial stewardship. Clin Infect Dis 2007; 44: 159-177.

(Received 16 Feb 2011, accepted 17 Feb 2011) 